\title{
Effect of Adding Metformin to Insulin Therapy in Pregnant Women with Uncontrolled Type I Diabetes as Regard Pregnancy Outcome
}

\section{Original Article}

\author{
Amal Kotb Abdullah', Amir A Gabr', Doaa Hamdy Hamed ${ }^{3}$, Mohamed A. Eweis ${ }^{4}$ \\ Department of Obstetrics and Gynecology, Faculty of Medicine, ${ }^{1,3,4}$ Beni-Suef University, \\ ${ }^{2}$ Cairo University, Egypt
}

\begin{abstract}
Aim: This study aimed to examine if addition of metformin to insulin is an effective alternative to insulin alone for women with type I Diabetes Mellitus to lower insulin dose, hospital stay, maternal and neonatal complications, particularly in a resource-poor setting.

Materials and Methods: Our randomized controlled trial included eighty patients, who were recruited into two equal groups; group A treated with metformin and insulin and group B treated with insulin alone, in Cairo and Beni-suef University Hospitals.

Results: We found that metformin plus insulin group (Group A) showed significant outcomes regarding shorted hospital stay, lower insulin dose and lower maternal weight gain ( $p$ values 0.04,0.02, 0.03, respectively). Most of maternal and neonatal complications were non-significantly lower in group A.

Conclusion: Oral metformin therapy is an effective and safe additional treatment option for women with type I diabetes during pregnancy to lower the insulin requirements, hospital stay and most maternal-fetal complications in settings with limited economic resources.
\end{abstract}

Key Words: GDM, maternal complications of type I diabetes mellitus, metformin in pregnancy, neonatal complications, type I diabetes mellitus

Received: 27 June 2021, Accepted: 17 July 2021

Corresponding Author: Mohamed A. Eweis, Department of Obstetrics and Gynecology, Faculty of Medicine, Beni-Suef University, Tel.: : +20822318605, E-mail: medmohamd@med.bsu.edu.eg

ISSN: 2090-7265, August 2021, Vol.11, No. 3

\section{INTRODUCTION}

Gestational diabetes mellitus (GDM) is known as glucose intolerance first diagnosed or developed during pregnancy 1 . Obesity, family history of diabetes, increased maternal age, past history of GDM and fetal macrosomia are risk factors for developing GDM 2. Both maternal and fetal adverse effects include preeclampsia, cesarean section, fetal macrosomia, neonatal hypoglycemia, hyperbilirubinemia, birth trauma, death, and obesity or diabetes later in life. Also half of these mothers will develop type $2 \mathrm{DM}$ and/or GDM later in life $\mathrm{e}^{[1,2]}$.

Current methods of glycemic control include lifestyle modification, self-monitoring of blood glucose (SMBG) and pharmacological agents such as insulin, Metformin and Glypuride ${ }^{[3]}$. Many randomized controlled trials and observational studies compared Metformin with insulin and glyburide ${ }^{[4]}$. After insulin, Metformin may be considered the preferred first-line option when an oral agent is chosen by the patient or provider as it has better fetal outcome compared to Glyburide.

Many studies showed that Metformin has beneficial effects as lower maternal weight gain and lower risk of pregnancy induced hypertension (PIH). Other benefits include a lower rate of maternal hypoglycemia than insulin or glyburide, lower cost and less need for SMBG. However, its association with increased rate of preterm deliveries was debatable among different studies. Two meta-analyses of studies for continued use of Metformin during pregnancy in patients with polycystic ovary syndrome (PCOS) showed a lower risk of abortion and preterm delivery with Metformin ${ }^{[5,6]}$.

Recent meta-analyses and other studies did not report any increase in preterm deliveries with the use of Metformin ${ }^{[7-9]}$. Another meta-analysis comparing Metformin versus Glyburide, Metformin was associated with lower rates of neonatal death, while glyburide was associated with increased risk of neonatal hypoglycemia, higher birth weight and greater incidence of macrosomia and higher maternal weight gain ${ }^{[10]}$. Other meta-analysis demonstrated the effect of Metformin on reducing the risk of preeclampsia, macrosomia, large for gestational age (LGA), neonatal hypoglycemia and NICU admission ${ }^{[8]}$.

Also, Metformin was better than insulin or glyburide as regards macrosomia, PIH, LGA and respiratory distress, 
but has highest rate of preterm birth in obese GDM women ${ }^{[11]}$.

ACOG recommends that insulin be the preferred treatment if glycemic control is not achieved by nonpharmacologic methods. If a patient cannot take insulin or declines, Metformin can be used after counselling the patient about the possible risks ${ }^{[12]}$. A systematic review and meta-analysis by Bao et al., reported that Metformin may have potential maternal, fetal or neonatal benefits with no major adverse effects in $\mathrm{GDM}^{[13,14]}$. However, more studies are required to provide more evidence for the use of Metformin for type I DM during pregnancy. Regarding insulin, the cost and system of parenteral dosing, it may not be appropriate in settings with limited resources, patient illiteracy and insulin lacking. The cost and multiple parenteral administration represent a problem. In addition, the risk of hypoglycemia and maternal weight gain are undesirable effects. The oral route of administration, low cost and excellent patient compliance makes Metformin superior ${ }^{[15]}$. Moreover, the evidence is lacking as regards the effect of the combined Metformin and insulin therapy use on pregnancy outcomes in type I DM. This raised the need to perform this randomized controlled study.

\section{AIM OF THE WORK}

In our study, we aim to show the effect of adding metformin to insulin therapy in pregnant women with uncontrolled type I Diabetes.

\section{PATIENTS AND METHODS}

This randomized controlled clinical trial was conducted in Department of Obstetrics and Gynecology, Faculty of Medicine, Cairo University, Department of Obstetrics and Gynecology, Beni-Suef University Hospital, BeniSuef, Egypt. A total of 80 uncontrolled diabetic pregnant patients in the $3^{\text {rd }}$ trimester seeking medical advice and met the eligibility criteria, were asked about participation in the trial and given information pamphlets describing the study. Patients who agreed to join the trial gave written informed consent. The study was approved by the Ethics Committee. The RCT registration ID is NCT03928340 on $20^{\text {th }}$ April 2019.

\section{Inclusion and exclusion criteria:}

We included pregnant patients with type one diabetes and no other chronic disorders, on insulin therapy since start of gestation, singleton pregnancy with no apparent congenital anomalies and $\mathrm{HbAlc}$ level between 7\% to $11 \%$. Patients with type 2 or gestational diabetes, intolerance or hypersensitivity to Metformin, congestive heart failure or a history of congestive heart failure, renal or hepatic insufficiency, severe vomiting requiring intravenous fluids or hospitalization, diabetic ketoacidosis, a history of diabetic ketoacidosis or history of lactic acidosis higher order pregnancies (twins, triplets, etc.) and fetal lethal anomaly were excluded. Masking was followed using envelopes which were numbered serially and in each envelope the corresponding letter which detect the targeted group (A or B) was put according to computer based randomization then all envelops were closed and put in one box.

\section{Intervention:}

The patients were assigned to one of 2 groups: Group A (study group) included 40 patients who were treated with Metformin (as Cidophage $500 \mathrm{mg}$, CID Pharmaceuticals, Cairo, Egypt) given at a dose of $2000 \mathrm{mg}$, divided into two doses, taken with the 2 main meals, in addition to previous adjusted insulin dose. Insulin therapy was given subcutaneous by insulin syringes, the dose was calculated as 0.7 to 1.0 units $/ \mathrm{kg} / \mathrm{d}$, the total dose was given in two divided doses of a mixture of regular insulin and neutral protamine Hagedorn insulin (NPH) at a ratio of $3: 7,100 \mathrm{IU} / \mathrm{ml}$ (Insulin Mixtard 70/30, Novo Nordisk, Bagsværd, Denmark): two-thirds in the early morning (7-8 am) and one third in the evening $(5-7 \mathrm{pm})^{[16]}$. Glycemic response to Metformin treatment was assessed by checking fasting and 2-h postprandial blood glucose 5 days after the treatment was started. Group B (control group) included 40 patients who were treated with insulin alone, as described before (Insulin therapy was given subcutaneous by insulin syringes, the dose was calculated as 0.7 to 1.0 units $/ \mathrm{kg} / \mathrm{d}$, the total dose was given in two divided doses of a mixture of regular insulin and neutral protamine Hagedorn insulin (NPH) at a ratio of 3:7, $100 \mathrm{IU} / \mathrm{ml}$ (Insulin Mixtard 70/30, Novo Nordisk, Bagsværd, Denmark): twothirds in the early morning (7-8 am) and one third in the evening $(5-7 \mathrm{pm})^{[16]}$. Poor glycemic control was defined as fasting blood glucose $[95 \mathrm{mg} / \mathrm{dl}$ and/or 2 -h postprandial blood glucose $120 \mathrm{mg} / \mathrm{dl}]^{[17]}$.

All patients underwent full history taking, thorough clinical examination, fundus examination at Ophthalmology clinic, laboratory investigations; $\mathrm{CBC}, \mathrm{HbA1c}$, coagulation profile, liver and kidney function at time of participation, at 28-32 weeks. FBS, $2 \mathrm{~h}$ postprandial blood sugar were done weekly till time of delivery.

Also, follow up included daily fetal kick count, weekly fetal weight gain, maternal weight gain, fundal level in relation to period of amenorrhea, CTG and fetal ultrasound. Subsequent management for uncontrolled cases were followed up weekly in the High Risk Pregnancy outpatient clinic until 36 weeks and then admitted to High Risk Pregnancy department for termination of pregnancy.

Resistant uncontrolled cases were admitted immediately to the High Risk Pregnancy department where capillary blood sugar was measured 7 times daily. Inpatient follow up included daily fetal surveillance by fetal kick count, CTG and regular fetal ultrasound every 3 days. 
At time of termination, assessment is done by fasting blood sugar, 2 hours postprandial blood sugar and HBA1C in addition to the routine preoperative labs. Also assessment included estimated fetal weight and maternal weight. Neonatal assessment after delivery include APGAR score, neonatal weight, incidence of transient tachypnea of newborn (TTN), respiratory distress syndrome (RDS), neonatal hypoglycemia and NICU admission.

\section{Outcomes:}

The primary outcome measures were $\mathrm{HbAlc}$, fasting blood sugar (after fasting for 8 hours), 2 hours postprandial blood sugar. Secondary Outcome Measures were maternal weight gain in kilograms, weekly estimated fetal weight gain by ultrasound, the dose of insulin taken by patient, attacks of maternal hypoglycemia (plasma glucose level below $65 \mathrm{mg} / \mathrm{dl}$ ), intra uterine fetal death (IUFD), neonatal weight in kilograms, preterm birth, neonatal respiratory distress, neonatal hypoglycemia (plasma glucose level below $45 \mathrm{mg} / \mathrm{dL}(2.5 \mathrm{mmol} / \mathrm{L}))$, neonatal Intensive care unit (NICU) admission.

\section{Sample size:}

Sample size calculation was done using the comparison of $\mathrm{HbA} 1 \mathrm{c}$ level between pregnant methods with type I DM treated with insulin alone and those treated with insulin plus metformin, as it was the primary outcome of our study. As reported in previous publication15.the mean \pm SD of $\mathrm{HbA} 1 \mathrm{c}$ in diabetic pregnancy treated with insulin was approximately $5.7 \pm 0.6 \%$, and we assumed that addition of metformin will achieve at least $10 \%$ improvement in HbAlc. Accordingly, we calculated that the minimum proper sample size was 24 participants in each group to achieve $80 \%$ power at $\alpha=0.05$ level using Student's t test for independent samples. Sample size calculation was done using Stats Direct statistical software version 2.7.2 for MS Windows, Stats Direct Ltd., Cheshire, UK.

\section{STATISTICAL ANALYSIS:}

The data was collected on Microsoft Excel Sheet 2010. Data were statistically described in terms of mean \pm standard deviation $( \pm \mathrm{SD}$ ) or frequencies (number of cases) and percentages when appropriate. Comparison of numerical variables between the study groups was done using Mann Whitney $\mathrm{U}$ test for independent samples. For comparing categorical data, Chi-square $( \pm 2)$ test was performed. Exact test was used instead when the expected frequency is less than 5. p values less than 0.05 were considered statistically significant using computer program IBM SPSS (Statistical Package for the Social Science; IBM Corp, Armonk, NY, USA) release 22 for Microsoft Windows.

\section{RESULTS}

Eighty pregnant females participated in the study divided into two groups. Table I shows the demographic characteristics and antenatal follow up criteria in the studied groups, there were no significant difference in the baseline terms as age, education, profession and BMI $p$-value $>.05$.

The duration of DM (in years) was nonsignificantly higher in group A than in group $\mathrm{B}(17.18 \pm 7.66,15.73 \pm 5.65, \rho 0.34)$. No significant difference between the two groups regarding the resistant uncontrolled cases who were admitted to high risk pregnancy department or pregnancy associated hypertensive disorders.

As regards the change in insulin dose, the increase in insulin dose was $30 \%$ and $80 \%$ (p 0.03), while $10 \%, 0 \% \quad(p<0.001)$ has decreased dose and $60 \%, 20 \%(p<0.001)$ did not change the dose of insulin in Metformin and insulin only groups, respectively.

The number of hypoglycemic attacks were non-significantly lower in group $\mathrm{A}$ than in group B $(0.18 \pm 0.55, \quad 0.50 \pm 1.04, \quad \rho 0.84)$. Severe preeclampsia developed in $1(2.5 \%)$ of cases, and $2(5 \%)$ in group A and group B, respectively $(p 0.55)$, while mild cases were equal in both groups $3(7.5 \%)$.

At time of termination, the estimated fetal weight by ultrasound was similar in group $\mathrm{A}$ and group B $(3,426.15 \pm 548.89,3,411.32 \pm 437.50, \rho 0.9)$. The AFI was non-significantly lower in group A than in group B $(15.62 \pm 5.76,16.79 \pm 5.69, \rho 0.37)$. The Resistance index (RI) of umbilical artery Doppler was non-significantly higher in group $\mathrm{A}$ than in group B $(0.60 \pm 0.08,0.59 \pm 0.05, \rho 0.45)$. Table 2 shows intra-partum, immediate postpartum care criteria and neonatal complications in the studied groups. 
Table 1: Demographic and antenatal follow up criteria in the studied groups

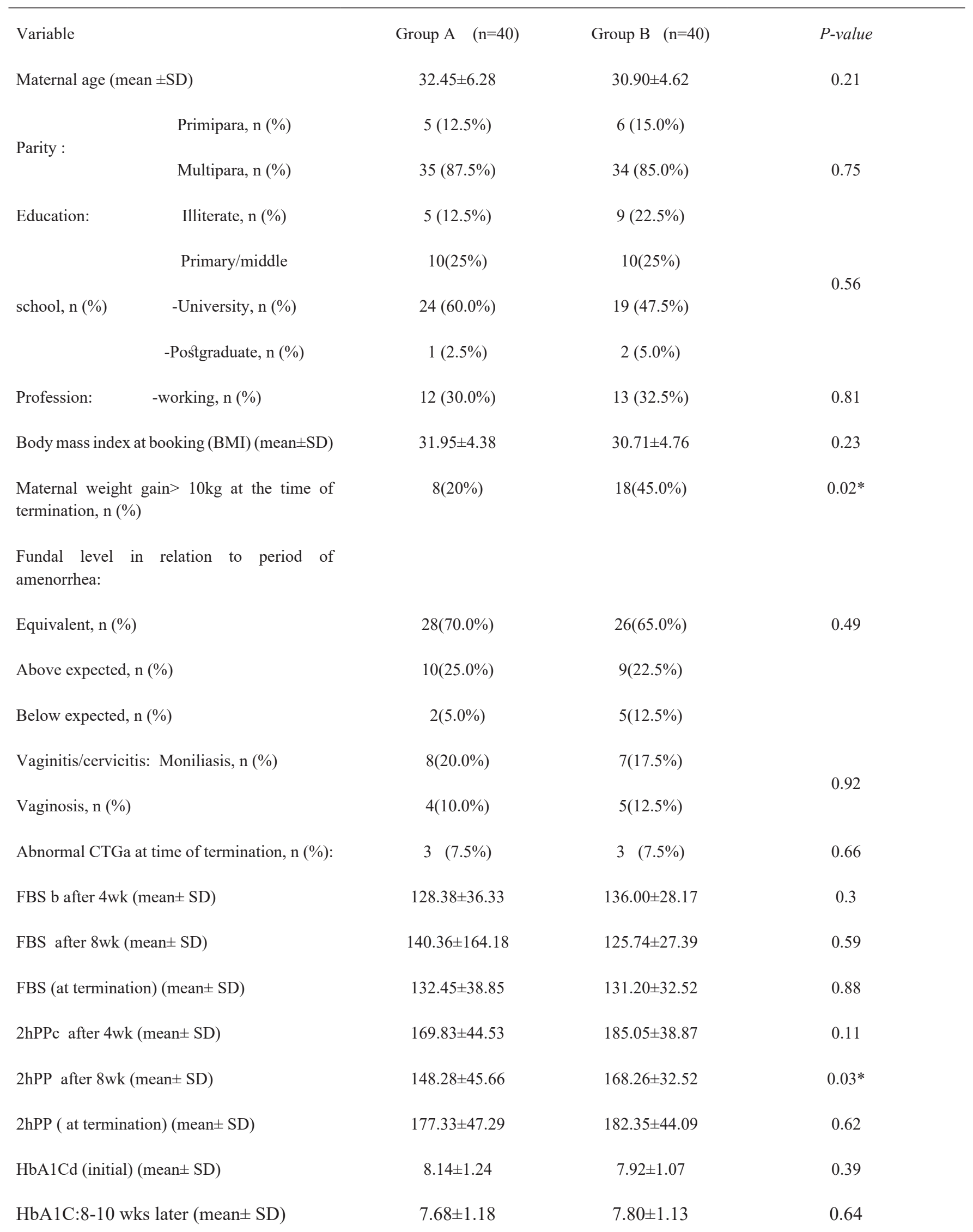


weeks:

equivalent, $\mathrm{n}(\%)$

-Above, n (\%)

Below, n (\%)

Follow up of fetal growth by Ultrasound: at 32weeks:

-equivalent, n (\%)

-Above, n (\%)

-Below, n (\%)

Follow up of fetal growth by Ultrasound: at 36 weeks:

-equivalent, n (\%)

-Above, n (\%)

-Below, n (\%)
$36(90.0 \%)$

$4(10.0 \%)$

$0(00.0 \%)$

$28(70.0 \%)$

$9(22.5 \%)$

$3(7.5 \%)$
0.23

$10(25.0 \%)$

$4(10.0 \%)$

Total (38 cases) Total (39 cases)

$25(65.8 \%) \quad 25(64.1 \%)$

0.37

Table 2: Intra-partum, immediate postpartum criteria and neonatal complications in the studied groups

$12(31.6 \%) \quad 10(25.6 \%)$

$1(2.6 \%)$

$4(10.3 \%)$

\begin{tabular}{|c|c|c|c|}
\hline Variable & GroupA $(n=40)$ & GroupB $(\mathrm{n}=40)$ & $p$-value \\
\hline \multicolumn{4}{|l|}{ Time of termination in weeks: } \\
\hline$\leq 39$ weeks $(\mathrm{n}, \%)$ & $32(80 \%)$ & $24(60 \%)$ & 0.05 \\
\hline$>39$ weeks $(\mathrm{n}, \%)$ & $8(20 \%)$ & $16(40 \%)$ & \\
\hline \multicolumn{4}{|l|}{ Mode of delivery: } \\
\hline Vaginal delivery, n (\%) & $8 \quad(20.0 \%)$ & $9(22.5 \%)$ & 0.79 \\
\hline Caesarean section, $\mathrm{n}(\%)$ & $32(80.0 \%)$ & $31(77.5 \%)$ & \\
\hline Preterm labour:, n (\%) & $2(5.0 \%)$ & $1(2.5 \%)$ & 0.56 \\
\hline Birth trauma:, n (\%) & $3(7.5 \%)$ & $1(2.5 \%)$ & 0.31 \\
\hline Neonatal Birth weight in grams (mean \pm SD) & $3,430.00 \pm 628.88$ & $3,433.75 \pm 515.20$ & 0.98 \\
\hline Apgar-1 (mean \pm SD) & $6.93 \pm 1.526$ & $6.90 \pm 1.566$ & 0.94 \\
\hline Apgar-5 $($ mean \pm SD) & $8.50 \pm 1.155$ & $8.33 \pm 1.403$ & 0.54 \\
\hline
\end{tabular}


Hospital stay in High risk pregnancy unit in $0.28 \pm 0.45$ days (mean $\pm \mathrm{SD})$

Neonatal hypoglycemia:, n (\%)

$16(40.0 \%)$

Transient tachypnea of newborn (TTN):n (\%) $10(25.0 \%)$

Respiratory Distress Syndrome (RDS): n(\%) 7(17.5\%)

NICU admission>24hours: $\mathrm{n}(\%)$

\section{$0.50 \pm 0.51$}

$0.04 *$

$9(22.5 \%)$

0.091

$7(17.5 \%)$

0.412

$3(7.5 \%)$

0.176

$11(27.5 \%)$

0.606

\section{DISCUSSION}

To our knowledge, there were limited data about combined use of Metformin and insulin for uncontrolled type I DM, during pregnancy. Eighty patients were randomized into 2 equal groups in this study. Demographic characteristics of patients in the Metformin and insulin only groups are nearly similar.

Metformin has more advantage than insulin as regards the oral route of administration, low cost and excellent patient compliance. When combined with insulin, the glycogenic control was tested in this study. The effect of Metformin on $\mathrm{HbAlc}$, fasting blood sugar, 2 hours postprandial blood sugar was not significant (except at 8 weeks, $p$ 0.03). However, it does not stimulate insulin production and is therefore not associated with the risk of hypoglycemia ${ }^{[18]}$.

Maternal weight gain in kilograms was significantly lower in Metformin group which agrees with other studies ${ }^{[9,19]}$. Whereas the estimated fetal weight gain by ultrasound was not significantly higher in Metformin group at 28 and 32 weeks and similar at 36 weeks.

A systematic review and meta-analysis by Bao et al. showed that Metformin alone lowered the risk of neonatal hypoglycemia, macrosomia, large for gestational age babies and neonatal intensive care unit admission. In our study, the combined use of Metformin and insulin was not significantly effective in lowering those risks ${ }^{[20]}$.

The increase in insulin dose was $30 \%$ and $80 \%$ in both groups ( $p 0.03)$, while $60 \%, 20 \%(p<0.001)$ did not change the dose of insulin in metformin and insulin only groups, respectively, which adds to the benefits of using combined metformin and insulin. These results are similar to other studies which show that $53.8 \%$ of the patients required insulin from their first visit due to 'overt' diabetes. In patients using oral hypoglycemic agents (OHAs), only $7.8 \%$ of cases needed to be switched from OHA to insulin $^{[19]}$

The time of termination of pregnancy was $\leq 39$ weeks in $80 \%$ of patients in the metformin group compared to $60 \%$ in insulin only group $(p 0.05)^{[19]}$.
The risk of developing preeclampsia in type I $\mathrm{DM}$ pregnant women is between $12 \%$ to $15 \%$, with increased risk (50\%) in pre-preexisting nephropathy ${ }^{[20-22]}$. Compared to our study, the development of severe preeclampsia was non-significantly lower in the metformin group $1(2.5 \%), 2(5 \%), p 0.55)$, while mild cases were equal in both groups $3(7.5 \%), 3(7.5 \%)$.

The addition of metformin to insulin did not increase the caesarean section rate, preterm birth or growth restriction which agrees with similar study ${ }^{[13]}$. However, opposing results are found in other studies ${ }^{[23-25]}$.

Oral metformin therapy is an effective and safe treatment option for women with type I diabetes during pregnancy to lower the insulin requirements and hospital stay and to lower most of maternal and neonatal complications. But further follow-up is needed to establish long-term safety regarding the pregnancy outcome. There were no serious adverse events associated with the use of metformin.

Long-term results were not included in the study due to difficulty to communicate with patients for follow up after labor. Future studies on larger sample of patients are needed to expand our understanding of the effect of combined metformin and insulin therapy in pregnancies complicated with type I DM.

The strength point in our study, we followed the consort statement of randomized study, the good sample size and the follow up of intervention with small dropout. Our limitation is the lack of data and evidence in the topic of our study.

\section{CONCLUSION}

Oral metformin therapy is an effective and safe treatment option for women with type I diabetes during pregnancy to lower the insulin requirements, hospital stay and most maternal and fetal complications in settings with limited economic resources.

\section{CONFLICT OF INTEREST}

There are no conflicts of interests. 


\section{REFERENCES}

1. Committee on Practice Bulletins-Obstetrics. Practice bulletin no. 137: gestational diabetes mellitus. Obstet Gynecol. 2013 Aug;122 (2 Pt 1):406-16.

2. American Diabetes Association. Standards of medical care in diabetes-2010. Diabetes care. 2010 Jan 1;33(Supplement 1):S11-61.

3. Falavigna M, Schmidt MI, Trujillo J, Alves LF, Wendland ER, Torloni MR, Colagiuri S, Duncan BB. Effectiveness of gestational diabetes treatment: a systematic review with quality of evidence assessment. Diabetes research and clinical practice. 2012 Dec 1;98(3):396-405.

4. Kelley KW, Carroll DG, Meyer A. A review of current treatment strategies for gestational diabetes mellitus. Drugs in context. 2015;4.

5. Feng L, Lin XF, Wan ZH, Hu D, Du YK. Efficacy of metformin on pregnancy complications in women with polycystic ovary syndrome: a metaanalysis. Gynecological Endocrinology. 2015 Nov 2;31(11):833-9.

6. Tan X, Li S, Chang Y, Fang C, Liu H, Zhang $X$, Wang Y. Effect of metformin treatment during pregnancy on women with PCOS: a systematic review and meta-analysis. Clinical and Investigative Medicine. 2016 Sep 11:E120-31.

7. Feng YE, Yang H. Metformin-a potentially effective drug for gestational diabetes mellitus: a systematic review and meta-analysis. The Journal of Maternal-Fetal \& Neonatal Medicine. 2017 Aug 3;30(15):1874-81.

8. Farrar D, Simmonds M, Bryant M, Sheldon TA, Tuffnell D, Golder S, Lawlor DA. Treatments for gestational diabetes: a systematic review and meta-analysis. BMJ open. 2017 Jun 1;7(6).

9. Priya G, Kalra S. Metformin in the management of diabetes during pregnancy and lactation. Drugs in context. 2018;7.

10. Brown J. Oral anti-diabetic pharmacological therapies for the treatment of women with gestational diabetes [Internet]. Cochrane Database of Systematic Reviews, Oxford, CD011967. 2017.
11. Liang HL, Ma SJ, Xiao YN, Tan HZ. Comparative efficacy and safety of oral antidiabetic drugs and insulin in treating gestational diabetes mellitus: an updated PRISMA-compliant network metaanalysis. Medicine. 2017 Sep;96(38).

12. Caughey AB, Turrentine M. Gestational diabetes mellitus. Obstetrics and Gynecology. 2018 Feb 1;131(2):E49-64.

13. Le-xin Bao, Wan-ting Shi, Yu-xin Han. Metformin versus insulin for gestational diabetes: a systematic review and meta-analysis, The Journal of Maternal-Fetal \& Neonatal Medicine, 2019, DOI: $10.1080 / 14767058.2019 .1670804$.

14. Dickens LT, Thomas CC. Updates in gestational diabetes prevalence, treatment, and health policy. Current diabetes reports. 2019 Jun 1;19(6):33.

15. Singh N, Madhu M, Vanamail P, Malik N, Kumar S. Efficacy of metformin in improving glycaemic control \& perinatal outcome in gestational diabetes mellitus: a non-randomized study. The Indian journal of medical research. 2017 May;145(5):623.

16. American College of Obstetricians and Gynecologists (ACOG): Gestational diabetes mellitus. Practice, Bulletin No. 180, July 2017a.

17. Pridjian G, Benjamin TD. Update on gestational diabetes. Obstetrics and Gynecology Clinics. 2010 Jun 1;37(2):255-67.

18. DeFronzo RA. Pharmacologic therapy for type 2 diabetes mellitus. Annals of internal medicine. 1999 Aug 17;131(4):281-303.

19. Nicolaou V, Soepnel L, Huddle KR, Levitt N, Klipstein-Grobusch K, Norris SA. Maternal and neonatal outcomes following the introduction of oral hypoglycaemic agents for gestational diabetes mellitus were comparable to insulin monotherapy in two historical cohorts. SAMJ: South African Medical Journal. 2020 Feb;110(2):154-8.

20. Sibai BM, Caritis SN, Hauth JC, MacPherson C, VanDorsten JP, Klebanoff M, Landon M, Paul RH, Meis PJ, Miodovnik M, Dombrowski MP. 
Preterm delivery in women with pregestational diabetes mellitus or chronic hypertension relative to women with uncomplicated pregnancies. American journal of obstetrics and gynecology. 2000 Dec 1;183(6):1520-4.

21. Reece EA, Eriksson UJ. Congenital malformations: epidemiology, pathogenesis, and experimental methods of induction and prevention. Diabetes in Women: Adolescent, Pregnancy, and Menopause. Lippincott Williams \& Wilkins, Philadelphia. 2004:169-204.

22. Vargas R, Repke JT, Ural SH. Type 1 diabetes mellitus and pregnancy. Reviews in obstetrics and gynecology. 2010;3(3):92.
23. Mariam L, Abeer BA, Hassan A, Rasha EK, Mahmoud A. Assessment of Metformin Versus Insulin for the Treatment of Gestational Diabetes. The Medical Journal of Cairo University. 2019 Jun 10;87(June):2385-92.

24. Rowan JA, Hague WM, Gao W, Battin MR, Moore MP. Metformin versus insulin for the treatment of gestational diabetes. New England Journal of Medicine. 2008 May 8;358(19):2003-15.

25. Niromanesh S, Alavi A, Sharbaf FR, Amjadi N, Moosavi S, Akbari S. Metformin compared with insulin in the management of gestational diabetes mellitus: a randomized clinical trial. Diabetes research and clinical practice. 2012 Dec 1;98(3):422-9. 